\title{
Search for Higgs boson production in association with a top-quark pair at CMS
}

\author{
Eleni Ntomari on behalf of the CMS Collaboration* \\ Deutsches Elektronen-Synchrotron (DESY) - Hamburg \\ E-mail: eleni.ntomari@cern.ch
}

Results of the search for the associated top-quark pair and Higgs-boson production $(t \bar{t} H)$ in proton-proton collisions with center-of-mass energy $\sqrt{s}=13 \mathrm{TeV}$ are presented. For the $t \bar{t} H(\gamma \gamma)$ and $t \bar{t} H$ (multileptons) the analyses are performed using the dataset recorded with the CMS experiment [1] in 2016, that corresponds to an integrated luminosity of $12.9 \mathrm{fb}^{-1}$, while for the $t \bar{t} H(b \bar{b})$, the integrated luminosity corresponds to $2.7 \mathrm{fb}^{-1}$ from the 2015 dataset. The $t \bar{t} H$ process allows a direct measurement of the top-Higgs coupling, which is an important test of the Standard Model (SM), and for searching new physics. Three statistically independent analyses are performed and optimized individually according to different Higgs boson decays, and in each of them, the results are obtained for the signal strength cross section ratio of the measurement to the SM prediction, $\hat{\mu}=\sigma / \sigma_{S M}$. The decay to bottom quark-antiquark pair, $t \bar{t} H(b \bar{b})$, results in $\hat{\mu}_{o b s}^{t \bar{t} H(b \bar{b})}=-2.0_{-1.8}^{+1.8}$, the diphoton decay, $t \bar{t} H(\gamma \gamma)$, in $\hat{\mu}_{o b s}^{t \bar{t} H(\gamma \gamma)}=1.9_{-1.2}^{+1.5}$, and the decay to multileptons, $t \bar{t} H$ (multileptons), results in $\hat{\mu}_{o b s}^{t \bar{t} H(\text { multileptons })}=2.3_{-0.8}^{+0.9}$. A combined result of $\hat{\mu}_{o b s}^{t \bar{t} H}=0.15_{-0.81}^{+0.95}$ obtained with the 2015 dataset is compatible with the SM expectation.

38th International Conference on High Energy Physics

3-10 August 2016

Chicago, USA

${ }^{*}$ Speaker. 


\section{Introduction}

The LHC Run I data produced at the Large Hadron Collider (LHC) by proton-proton collisions at center-of-mass energies of $\sqrt{s}=7 \mathrm{TeV}$ and $8 \mathrm{TeV}$, have been exploited by both ATLAS and CMS collaborations, to measure nearly all the accessible properties of the newly-discovered Higgs boson in 2012, with a mass of approximately $125 \mathrm{GeV}$ [2,3]. After the discovery, the focus lies on measuring its properties in order to find out if the Higgs boson is as predicted by the Standard Model (SM), and irrespective of this, if it reveals any signs of new physics beyond the SM (BSM).

One central aspect to answer these questions is to measure the coupling constants of the boson to the other elementary particles, which according to the SM, depend on the mass. The top quark, as the heaviest elementary particle, has the strongest coupling of all. Indirect constraints on the topquark Higgs boson coupling are available from processes including top-quark loops, for example Higgs boson production through gluon-gluon fusion that is the largest contribution to the total SM Higgs production cross section at the LHC. On the other hand, the top-Higgs interaction vertex is directly accessible when the Higgs boson is produced in association with one or more top-quarks, since the Higgs boson is too light to decay to top-quarks directly. The associated production with a single top-quark is suppressed in the SM, while the associated production of a Higgs boson and a top quark-antiquark pair ( $(\bar{t} H$ production) offers the best opportunity for a direct measurement of its magnitude at the LHC in the SM framework.

This report presents the first $t \bar{t} H$ results from CMS at $\sqrt{s}=13 \mathrm{TeV}$ using the 2016 dataset for $t \bar{t} H$ (multileptons) and $t \bar{t} H(\gamma \gamma)$, and the 2015 dataset for the $t \bar{t} H(b \bar{b})$. A summary of the results with 7 and $8 \mathrm{TeV}$ data (Run I) can be found in Ref. [4] and corresponds to an observed (expected) significance of $4.4 \sigma(2.0 \sigma)$ over the null hypothesis.

\section{$1.1 t \bar{t} H(b \bar{b})$}

The Higgs boson decay into bottom quark-antiquark pairs $(b \bar{b})$ is attractive as a final state because it features the largest branching fraction of $0.58 \pm 0.02$ for a $125 \mathrm{GeV}$ Higgs boson. The main challenge comes from the overwhelming background from $t \bar{t}+$ jets. The main strategy is to constrain the backgrounds and obtain a good signal separation and is done by forming many orthogonal categories. The two main categories are defined according to the lepton multiplicity of the $t \bar{t}$ system decay: "lepton+jets" with exactly one lepton and "dilepton" with exactly two opposite sign leptons. In order to improve the sensitivity of the analysis, events are divided into subcategories based on the number of jets and b-tags, and the presence of boosted jets from hadronic decays of top-quarks or $H \rightarrow b \bar{b}$ decays with large transverse momenta; the "boosted" subcategory is new with respect to Run I analysis. Boosted decision trees (BDTs) with different variables and a matrix element method (MEM) are used to achieve the optimal signal separation, in each of the 13 orthogonal subcategories.

Results are obtained from a simultaneous binned maximum-likelihood fit to data in all subcategories and can be seen in Fig.1 (left). The integrated luminosity of the analyzed data corresponds to $2.7 \mathrm{fb}^{-1}$. The combined best-fit value of the signal strength is $\hat{\mu}_{o b s}^{t \bar{t} H(b \bar{b})}=-2.0 \pm 1.8$ that is $1.7 \sigma$ below the SM expectation and thus compatible with the no signal assumption [6]. Since this analysis is systematics dominated, more precise results can be achieved with improvements in theory and 
modeling as well as with increase of statistics, since it will help to constrain several uncertainties and to understand the backgrounds better.
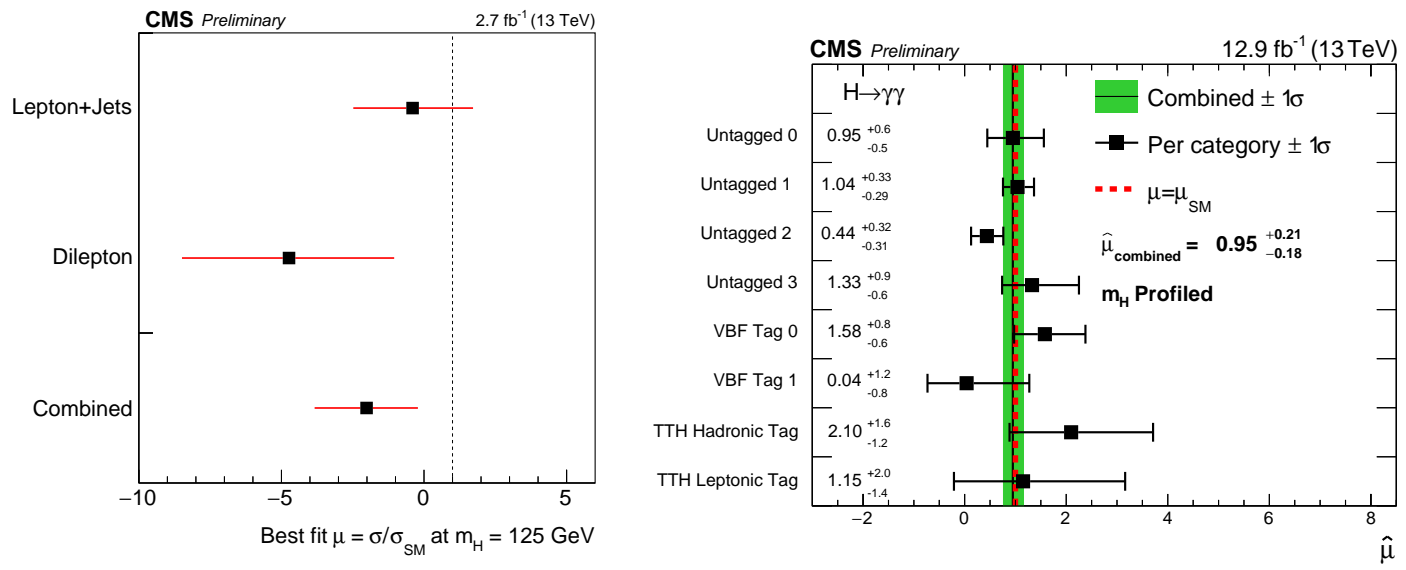

Figure 1: Left: Best fit values of the signal strength modifiers $\mu$ with their $\pm 1 \sigma$ confidence intervals for the $t \bar{t} H(b \bar{b})$ analysis. Right: Signal strength modifiers measured in each category (black points) for profiled $\mathrm{m}_{H}$, compared to the overall signal strength (green band) and to the SM expectation (dashed red line) for the $H \rightarrow \gamma \gamma$ analysis.

\section{$1.2 t \bar{t} H(\gamma \gamma)$}

Despite the small branching ratio predicted by the SM $(\sim 0.2 \%)$, the $H \rightarrow \gamma \gamma$ decay channel provides a clean final state with an invariant mass peak that can be reconstructed with great precision due to the excellent photon identification and energy resolution.

The analysis is an integral part of the $H \rightarrow \gamma \gamma$ analysis [7], where events are categorized according to production mode topologies(Vector Boson Fusion, VBF, and in association with top-quarks, $t \bar{t} H$ ). Those produced via $t \bar{t} H$ are accompanied by two b-quarks and two $W$ bosons, and may be accompanied by charged leptons or additional jets. The analysis is performed with $12.9 \mathrm{fb}^{-1}$ of data preselected with diphoton triggers.

The main backgrounds stem from the irreducible $t \bar{t}+\gamma \gamma$ and the reducible $t \bar{t}+\gamma+$ jet and the $t \bar{t}+$ jets where the jets are mis-identified as isolated photons. The main challenges here are the suppression of the mis-identified (fake) photons and corresponding backgrounds and to obtain an excellent diphoton mass resolution. Events are selected with leptons (at least one) from leptonic or semi-leptonic top decays, forming the "leptonic" category, and jets from hadronic top decays, forming the "hadronic" category (no leptons at all). Moreover there are requirements on the number of jets and b-tags. BDTs are used for photon identification and event selection. The event interpretation including signal and background models is identical with the $H \rightarrow \gamma \gamma$ analysis, searching for a diphoton mass resonance assuming a non-resonant background.

The observed signal strength for the combination of the two categories is $\hat{\mu}_{o b s}^{t \bar{t} H(\gamma \gamma)}=3.8_{-3.6}^{+4.5}$, estimated for $m_{H}=125.09 \mathrm{GeV}$, and is in agreement with the SM within the large uncertainties. The results are statistically limited and thus better precision is expected with increase of statistics. 


\section{$1.3 t \bar{t} H$ (multileptons)}

A third analysis, complementary to $t \bar{t} H(b \bar{b})$ and $t \bar{t} H(\gamma \gamma)$, targets leptonic final states from Higgs boson decays to $W W^{*}, Z Z^{*}$, or $\tau \tau$, with at least one $Z, W$, or $\tau$ decaying leptonically [9]. Despite the small branching ratio, the presence of one or two additional leptons from top-quark decays, leads to clean experimental signatures: two same-sign leptons or at least three leptons (electrons or muons) produced together with b-tagged jets. Since it has the smallest irreducible backgrounds from $t \bar{t}$ associated with vector bosons, $t \bar{t} W$ and $t \bar{t} Z$, the focus lies on understanding the complex reducible background $t \bar{t}+$ jets with non-prompt leptons mis-identified as prompt. The irreducible backgrounds are taken directly from simulation, while the reducible is estimated with data-driven methods. The general strategy lies on identification, with high efficiency, of prompt leptons and separation from non-prompt and charge mis-identified leptons.

The dataset used for this analysis corresponds to an integrated luminosity of $12.9 \mathrm{fb}^{-1}$. The events are selected by requiring the presence of either one, two, or three leptons (electrons or muons) at trigger level and are split in two main categories: the "dilepton" that requires exactly 2 same-sign leptons and the "trilepton" that requires at least 3 leptons. The $t \bar{t}$ signature is enhanced by requiring jets and b-tags. The analysis strategy is optimized for each category, but to enhance the sensitivity, the events are further sub-categorized according to different background compositions based on lepton charge, the presence of hadronically-decaying $\tau$ leptons, the lepton flavour and the presence of at least $2 \mathrm{~b}$-tags. In order to further improve the sensitivity of the analysis, topological and kinematic differences between signal and background events are exploited by means of BDTs and MEM, aimed at discriminating the signal from the $t \bar{t}$ and $t \bar{t} V$ processes respectively.

Table 1: Asymptotic 95\% CL upper limits and the best fit of the signal strength parameter.

\begin{tabular}{lccc}
\hline \hline Category & Observed UL & Expected UL $\pm 1 \sigma$ & $\mu$ best fit $\pm 1 \sigma$ \\
\hline same-sign dileptons & 4.6 & $1.7_{-0.5}^{+0.9}$ & $2.7_{-1.0}^{+1.1}$ \\
trileptons & 3.7 & $2.3_{-0.7}^{+1.2}$ & $1.3_{-1.0}^{+1.2}$ \\
\hline combined & 3.9 & $1.4_{-0.4}^{+0.7}$ & $2.3_{-0.8}^{+0.9}$ \\
combined with 2015 data & 3.4 & $1.3_{-0.4}^{+0.6}$ & $2.0_{-0.7}^{+0.8}$ \\
\hline \hline
\end{tabular}

The event yields are compared with the expectation from the background processes and a $125 \mathrm{GeV}$ SM Higgs boson. Checking the asymptotic 95\% CL upper limits on the signal strength parameter (Table 1), there is no significant excess.

\section{Conclusions}

First results of SM $t \bar{t} H$ production searches, using pp collision data collected by the CMS experiment in 2016 (and 2015 for $t \bar{t} H(b \bar{b})$ ) at center-of-mass energy of $\sqrt{s}=13 \mathrm{TeV}$, has been presented. Independent analyses are performed according to different Higgs decays in $t \bar{t} H(b \bar{b})$, $t \bar{t} H(\gamma \gamma)$ and $t \bar{t} H$ (multileptons), each of them using a separate analysis strategy. Combination of these three analyses, when using the 2015 dataset $[6,8,10]$, yields a signal strength of $\hat{\mu}_{o b s}^{t t h}=$ $0.15_{-0.81}^{+0.95}$, that is also in agreement with the SM [11]. 


\section{References}

[1] CMS Collaboration, "The CMS experiment at the CERN LHC“, JINST 3 S08004 (2008).

[2] ATLAS Collaboration, "Observation of a new particle in the search for the Standard Model Higgs boson with the ATLAS detector at the LHC“, Phys. Lett. B 716, 1 (2012).

[3] CMS Collaboration, "Observation of a new boson at a mass of $125 \mathrm{GeV}$ with the CMS experiment at the LHC“, Phys. Lett. B 716, 30 (2012).

[4] ATLAS and CMS Collaborations, "Measurements of the Higgs boson production and decay rates and constraints on its couplings from a combined ATLAS and CMS analysis of the LHC pp collision data at $\sqrt{s}=7$ and $8 \mathrm{TeV}^{*}$, ATLAS-CONF-2015-044, CMS-PAS-HIG-15-002.

[5] CMS Collaboration, " Measurement of $t \bar{t}$ production with additional jet activity, including $b$ quark jets, in the dilepton decay channel using pp collisions at $\sqrt{s}=8 \mathrm{TeV}$ “, Eur. Phys. J. C. 76 (2016) 379.

[6] CMS Collaboration, "Search for $t \bar{t} H$ production in the $H \rightarrow b \bar{b}$ decay channel with $\sqrt{s}=13 \mathrm{TeV} p p$ collisions at the CMS experiment.", CMS-PAS-HIG-16-004.

[7] CMS Collaboration, "Updated measurements of Higgs boson production in the diphoton decay channel at $\sqrt{s}=13 \mathrm{TeV}$ in pp collisions at CMS.", CMS-PAS-HIG-16-020.

[8] CMS Collaboration, "First measurements of the Higgs boson production in the diphoton decay channel at $\sqrt{s}=13 \mathrm{TeV}$ ", CMS-PAS-HIG-15-005.

[9] CMS Collaboration, "Search for associated production of Higgs bosons and top quarks in multilepton final states at $\sqrt{s}=13$ TeV.", CMS-PAS-HIG-16-022.

[10] CMS Collaboration, "Search for $t \bar{t} H$ production in multilepton final states at $\sqrt{s}=13 \mathrm{TeV}$ ", CMS-PAS-HIG-15-008.

[11] CMS Collaboration, https://twiki.cern.ch/twiki/bin/view/CMSPublic/TTHCombMoriond2016. 